\title{
Immune checkpoint targeting as anti-cancer immunotherapy: promises, questions, challenges and the need for predictive biomarkers at ASCO 2015
}

\author{
Hardev Pandha ${ }^{1} \cdot$ Graham Pawelec $^{2}$
}

Received: 15 July 2015 / Accepted: 28 July 2015 / Published online: 13 August 2015

(C) Springer-Verlag Berlin Heidelberg 2015

\begin{abstract}
Immunotherapy targeting "immune checkpoints" first made the headlines at the ASCO (American Society of Clinical Oncology) Annual Meeting in 2013, took centre stage at 2014 and consolidated its position as a potentially curative first-line therapy as reflected by the presentations at ASCO 2015. For the first time, previously refractory cancers are proving amenable to treatment, but still only a fraction, usually a minority, of patients respond. The hunt for factors predicting responses and for biomarkers to monitor treatment was a major theme of this year's meeting, as briefly discussed in this Editorial.
\end{abstract}

Keywords Immune checkpoint · Anti-CTLA-4 · PD-1 · PD-L1 · Biomarkers · ASCO 2015

$\begin{array}{ll}\text { Abbreviations } \\ \text { ASCO } & \text { American Society of Clinical Oncology } \\ \text { CRC } & \text { Colorectal cancer } \\ \text { MMR } & \text { Mismatch-repair } \\ \text { NSCLC } & \text { Non-small-cell lung cancer } \\ \text { TIM3 } & \text { T cell immunoglobulin domain and mucin } \\ & \text { domain } 3\end{array}$

The resurgence of cancer immunotherapy continues to gain momentum with the recent explosion of compelling clinical

Hardev Pandha

h.pandha@surrey.ac.uk

1 Section of Oncology, School of Biosciences, University of Surrey and Surrey Cancer Research Institute, Legget Building, Daphne Jackson Rd, Guildford GU2 7WG, UK

2 Second Department of Internal Medicine, Centre for Medical Research (ZMF), University of Tübingen, Tübingen, Germany trial outcome data showing efficacy across a range of malignancies previously thought to be resistant to immune modulation. This reflects the work of at least two decades and the unravelling of complex regulatory pathways involved in the immune responses to cancer. Specifically, there has been a great deal of progress in our understanding of how $\mathrm{T}$ cell activation is controlled through "immune checkpoints" for the generation of adaptive immune responses to malignancy, and of inhibiting these interactions to achieve significant and clinically meaningful antitumour effects $[1$, 2]. Currently (July 2015), the largest group of immunotherapy trials of the total of $>250$ registered at clinicaltrials. gov are of checkpoint inhibitors. The result to date is rapid clinical evaluation of inhibition of three molecules in particular: cytotoxic T lymphocyte antigen-4 (CTLA-4), programmed death-1 (PD-1) and programmed death ligand-1 (PD-L1). The clinical development of the first of these, the anti-CTLA-4 antibody ipilimumab, has posed considerable challenges, both in terms of understanding its mode of action, new nomenclature for many oncologists, identifying optimal dose and scheduling as well as introducing a new spectrum of toxicities in the clinic, some of which may be fatal. These issues have been addressed by educational programs and also the development of risk management algorithms. Thankfully, the anti-PD-1 agents appear to have lower toxicity profiles with lower rates of posttreatment interventions and hospital admissions (reviewed in $[3,4])$. The hypothetical synergy between combining anti-CTLA-4 therapy (targeting initiation of the immune response) and anti-PD-1 (targeting the effector phase) has been borne out by increased objective clinical responses but at the expense of increased toxicity and potentially prohibitive drug costs [5]. Combined with a large number of possible further targets at the immune checkpoint level, both antagonistic BTLA [B and T lymphocyte attenuator], 
VISTA [V-domain immunoglobulin suppressor of $\mathrm{T}$ cell activation], CD160, LAG3 [lymphocyte activation gene 3], TIM3 ([T-cell immunoglobulin domain and mucin domain $3]$, and CD244) and agonistic (4-1BB, OX40 ligand), there would appear to be huge potential for further progress. Arguably, the most significant progress this year, as manifest at the 2015 ASCO Annual Meeting in Chicago has been with anti-PD-1 targeting, reflecting the large number of agents developed against this molecule relative to CTLA-4. Tumours include renal, melanoma, liver, bladder, ovarian, and head and neck, and colorectal cancers, but perhaps the most significant findings have been in non-smallcell lung cancer (NSCLC) where the agent nivolumab was compared in a randomised phase III study to standard-of-care docetaxel chemotherapy and found to deliver increased progression-free and overall survival. This was a large trial of nearly 300 advanced non-squamous NSCLC patients in each arm in which higher expression of PD-L1 was found to be associated with better clinical responses (L Paz-Ares, Seville, Spain). The objective responses have been impressive in traditionally refractory cancers such as lung. However, puzzlingly, and unlike in melanoma, there appears to be a ceiling of response at around $30 \%$ with evidence of median duration of response of around 18 months (SJ Antonia, Tampa, FL). Clearly, predictive biomarkers would be of enormous value, but histological assessment of PD-L1 expression in routine pathology is fraught with difficulty related to the different antibodies used and the heterogeneity of ligand expression in the tumour and infiltrating cells (SN Gettinger, New Haven, CT). Even if PD-L1+ tumours are more likely to respond, there may be no difference in PFS (in melanoma; H. Kluger, New Haven, CT). Nonetheless, the main question posed here was whether checkpoint inhibitors will be the new standard of care for NSCLC, answered with a resounding "yes" even with imperfect predictive biomarkers. The next question was then whether such treatments can be moved out of specialised care facilities into the community. Here again, the answer seems to be "yes" according to the early results of a study on over 800 patients treated with PD-1 blockade (MD Hellmann, New York, NY).

Significantly, the evolution of these agents has not resulted in the identification of robust predictive biomarkers [6]. This is important in view of the potential toxicities these agents may induce, the limited proportion of patients they may benefit as well as the high costs. Selection of patients most likely to benefit from anti-PD-1 treatment is currently centred on the expression of the ligand, PD-L1, on tumour cells and infiltrating $\mathrm{T}$ cells in pre-treatment tissue. The landmark phase 1 study showed a high likelihood of response to anti-PD-1 if tissue biopsies expressed PD-L1 [7]. However, it has been difficult to use this form of tissue biomarker operationally as there is variability of read-out using different antibodies and the lack of true consensus regarding the threshold percentage positivity which would be deemed to be positive. This is currently set at $1 \%$, and is also further limited by paucity of tissue to assess as this may be a small core biopsy.

With the impressive clinical data in some patients, there is a need for further reflection as to the mechanisms underlying these responses and biomarkers correlating with them. Anti-CTLA-4 treatment improves the survival of patients with advanced-stage melanoma. However, although ipilimumab is now an approved treatment for patients with metastatic disease, it remains unclear exactly how it boosts tumour-specific T cell activity. Recently, it was shown that in melanoma patients anti-CTLA-4 treatment induced a significant number of newly detected $\mathrm{T}$ cell responses, but only infrequently boosted pre-existing immune responses. This provides strong evidence for anti-CTLA-4 therapy-enhanced $\mathrm{T}$ cell priming as a component of the clinical mode of action [8]. Furthermore, patients who derive a long-term benefit from ipilimumab appear to generate a neoantigen landscape that is specifically present in tumours with a strong response to CTLA-4 blockade (JD Wolchock, New York, NY). In addition, the determinants of response to PD-1 blockade appear to be shaped by the genomic alterations in tumour cells. Wholeexome sequencing of NSCLCs treated with pembrolizumab has shown that higher nonsynonymous mutation burdens in tumours were associated with improved objective responses, durable clinical benefit, and improved PFS. Efficacy also correlates with smoking-induced molecular signatures, higher neoantigen burden due to mutations and DNA repair pathway mutations [9]. Significantly, in colorectal cancer (CRC), observations that somatic mutations due to mismatch-repair (MMR) defects have the potential to encode "non-self" immunogenic antigens suggest that they may be more susceptible to immune checkpoint blockade. Thus, DT Le (Baltimore, MD) compared 25 CRC patients with MMR defects to 25 without, and found significantly better 20 -week PFS in the former under treatment with pembrolizumab. This was associated with an absence of TIL in the MMR-proficient patients and relatively low levels of PD-L1 expression, but abundant infiltrating cells in the MMR-deficient patients. Thus, MMR deficiency could be a predictive marker of responses to PD-1 blockade. However, only $4-5 \%$ of all tumours seem to manifest MMR deficiencies, so the fraction of patients that could be treated on the basis of this as a marker would be very low [10].

Although checkpoint inhibition results in impressive responses, these do not extend to the majority of patients and may be of quite limited duration. There is huge potential for synergy with existing cancer therapies which are capable of immune activation, as well as combined 
immunotherapies including the first licensed such agent, IL 2. This was illustrated by the report of abscopal effects in late-stage melanoma patients treated with intratumoral ipilimumab and IL 2 (C Bowen, Salt Lake City, UT). These combinations can be usefully classified into (1) modalities that enhance antigen presentation, such as radiation, cryotherapy, chemotherapy, targeted agents, vaccines, tolllike receptor (TLR) agonists, type I interferon and oncolytic viruses; (2) additional agents aiming to reverse $\mathrm{T}$ cell dysfunction, such as other immune checkpoint inhibitors; and (3) agents targeting other immune inhibitory mechanisms, such as inhibitors of indoleamine dioxygenase (IDO), regulatory $\mathrm{T}$ cells and myeloid-derived suppressor cells (MDSCs). For example, in the latter case, data were presented on the importance of MDSCs in predicting responses to nivolimumab in late-stage melanoma patients who had failed ipilimumab treatment (J Weber, Tampa, FL). This study of 126 patients revealed that MDSCs suppressed their $\mathrm{T}$ cell reactivity in vitro, which could be overcome by PD-1 blockade. Low levels of PD-L1 and Tim3 expression by the MDSCs correlated with responses and may have contributed to the clinical efficacy seen in this study. Ipilimumab itself may also act partly via its effects on MDSCs [11]. Both MDSCs and Tregs have been implicated in limiting anti-cancer immune responses in many contexts and are likely to be both predictive biomarkers as well as potential therapeutic targets [12]. Oncolytic viruses are emerging as ideal partners for checkpoint inhibitors through their induction of immunogenic tumour cell death. Their association with pro-inflammatory cytokine responses and importantly the induction of PD-L1 in both $\mathrm{T}$ cells and tumour cells through the expression of interferon $\gamma$. Already, a phase III intratumoral herpes simplex virus type 1 (HSV-1) study in metastatic melanoma has shown evidence of local immunogenicity, immune priming and abscopal effects in visceral metastases [13]. Combination of the same virus with ipilimumab is already underway, with evidence of marked synergy and high objective anti-tumour responses (A Ribas, Los Angeles, CA). There is a large number of oncolytic viruses in clinical development, both wild-type and those armed with immunostimulatory transgenes and virus-expressing libraries providing unique opportunities for disease-specific combinations.

This is a truly revolutionary time for cancer immunotherapy where we can now finally discuss radiological response and improved patient survival. Furthermore, responses have been observed even after initial responses followed by progression with one checkpoint inhibitor on switching to another. This suggests multiple opportunities for targeting based on the numerous molecules involved in T cell costimulation, either sequentially or contemporaneously. The second wave of this revolution has to include improvement in tissue and circulating biomarkers. Much information has been derived from the former, by examining tumourinfiltrating immune cells and establishing risk profiles [e.g. 14] or gene expression profiles in whole tumour biopsies or resected specimens (A Ribas, Los Angeles, CA), but sequential patient monitoring over the long-term would be more efficiently and cost-effectively accomplished by employing peripheral blood as an easily obtainable source. This raises many questions of its own concerning harmonisation and standardisation of assays to be employed, but these issues are under intensive investigation cooperatively in many centres [e.g. 15, 16]. This will allow more rapid development through patient selection, reduction in toxicity and make this form of cancer treatment more cost-effective.

\section{Compliance with ethical standards}

Conflicts of interest The authors declare that they have no conflicts of interest.

\section{References}

1. Ai M, Curran MA (2015) Immune checkpoint combinations from mouse to man. Cancer Immunol Immunother 64(7):885892. doi:10.1007/s00262-014-1650-8

2. Postow MA, Callahan MK, Wolchok JD (2015) Immune checkpoint blockade in cancer therapy. J Clin Oncol 33(17):1974-1982

3. Haanen JB, Hv Thienen, Blank CU (2015) Toxicity patterns with immunomodulating antibodies and their combinations. Semin Oncol 42(3):423-428. doi:10.1053/j.seminoncol.2015.02.011

4. Weber JS, Yang JC, Atkins MB, Disis ML (2015) Toxicities of immunotherapy for the practitioner. J Clin Oncol 33(18):20922099. doi:10.1200/JCO.2014.60.0379

5. Larkin J, Chiarion-Sileni V, Gonzalez R et al (2015) Combined nivolumab and ipilimumab or monotherapy in untreated melanoma. N Engl J Med 373(1):23-34. doi:10.1056/NEJMoa1504030

6. Awad MM, Hammerman PS (2015) Durable responses with PD1 inhibition in lung and kidney cancer and the ongoing search for predictive biomarkers. J Clin Oncol 33(18):1993-1994. doi:10.1200/JCO.2015.61.4172

7. Topalian SL, Hodi FS, Brahmer JR et al (2012) Safety, activity, and immune correlates of anti-PD1 antibody in cancer. N Engl $\mathrm{J}$ Med 366(26):2443-2454. doi:10.1056/NEJMoa1200690

8. Kvistborg P, Philips D, Kelderman S et al (2014) AntiCTLA4 therapy broadens the melanoma-reactive CD8+ T cell response. Sci Transl Med 6(254):254ra128. doi:10.1126/ scitranslmed.3008918

9. Rizvi NA, Hellmann MD, Snyder A et al (2015) Cancer immunology. Mutational landscape determines sensitivity to PD1 blockade in non-small cell lung cancer. Science 348(6230):124128. doi: $10.1126 /$ science.aaa 1348

10. Le DT, Uram JN, Wang H et al (2015) PD1 blockade in tumours with mismatch-repair deficiency. N Engl J Med 372(26):25092520. doi:10.1056/NEJMoa1500596

11. Pico de Coaña Y, Masucci G, Hansson J, Kiessling R (2014) Myeloid-derived suppressor cells and their role in CTLA4 blockade therapy. Cancer Immunol Immunother 63(9):977-983. doi:10.1007/s00262-014-1570-7

12. Castelli C, Rivoltini L, Rodolfo M, Tazzari M, Belgiovine C, Allavena P (2015) Modulation of the myeloid compartment of 
the immune system by angiogenic- and kinase inhibitor-targeted anti-cancer therapies. Cancer Immunol Immunother 64(1):8389. doi:10.1007/s00262-014-1576-1

13. Andtbacka RH, Kaufman HL, Collichio F et al (2015) Talimogene Laherparepvec improves durable response rate in patients with advanced melanoma. J Clin Oncol (pii: JCO.2014.58.3377 [Epub ahead of print])

14. Becht E, Goc J, Germain C, Giraldo NA, Dieu-Nosjean MC, Sautès-Fridman C, Fridman WH (2014) Shaping of an effective immune microenvironment to and by cancer cells.
Cancer Immunol Immunother 63(10):991-997. doi:10.1007/ s00262-014-1590-3

15. Chang S, Kohrt H, Maecker HT (2014) Monitoring the immune competence of cancer patients to predict outcome. Cancer Immunol Immunother 63(7):713-719. doi:10.1007/ s00262-014-1521-3

16. Santegoets SJ, Dijkgraaf EM, Battaglia A et al (2015) Monitoring regulatory $\mathrm{T}$ cells in clinical samples: consensus on an essential marker set and gating strategy for regulatory $\mathrm{T}$ cell analysis by flow cytometry. Cancer Immunol Immunother. [Epub ahead of print] 